\title{
Correlation of carotid artery disease and tinnitus: is it an auditory phantom in vascular surgery practice? A wide evidence-based review
}

\author{
Amr Abdelhaliem¹, Callum Howard², Mohamad Bashir' ${ }^{1}$, Hazem Elsantawy ${ }^{1}$, Haytham Al-Khaffaf' \\ 'Department of Vascular Surgery, Royal Blackburn Teaching Hospital, Blackburn BB2 3HH, UK. \\ ${ }^{2}$ Faculty of Biology, Medicine and Health, University of Manchester, Oxford Rd, Manchester M13 9PL, UK.
}

Correspondence to: Dr. Mohamad Bashir, Department of Vascular Surgery, Royal Blackburn Teaching Hospital, Haslingden Rd, Blackburn BB2 3HH, UK. E-mail: mohamad.bashir@nhs.net; Dr. Callum Howard, Faculty of Biology, Medicine and Health, University of Manchester, Manchester M13 9PR, UK. E-mail: callum.howard099@gmail.com

How to cite this article: Abdelhaliem A, Howard C, Bashir M, Elsantawy H, Al-Khaffaf H. Correlation of carotid artery disease and tinnitus: is it an auditory phantom in vascular surgery practice? A wide evidence-based review. Vessel Plus 2020;4:31.

http://dx.doi.org/10.20517/2574-1209.2020.13

Received: 1 May 2020 First Decision: 15 Jun 2020 Revised: 3 Aug 2020 Accepted: 12 Aug 2020 Published: 21 Oct 2020

Academic Editor: Mario F. L. Gaudino Copy Editor: Cai-Hong Wang Production Editor: Jing Yu

\begin{abstract}
Carotid artery diseases can result in many extracranial manifestations. Tinnitus is a recognised symptom and has long been correlated with significant internal carotid artery stenosis. It has been largely classified into pulsatile and non-pulsatile types with variable management approaches and prognoses. Surgical and endovascular approaches to treat carotid artery stenosis have not only aimed to reduce stroke rates but also to manage such symptoms. The clinical and cognitive evidence of such practices are broad and managed in a combined spectrum with otolaryngologists. This literature review aims to focus on current evidence and practice with vascular surgeons on the importance of dealing with tinnitus in managing carotid artery stenosis.
\end{abstract}

Keywords: Carotid artery disease, carotid artery stenosis, tinnitus, carotid endarterectomy

\section{INTRODUCTION}

Tinnitus is a common symptom affecting $10 \%-15 \%$ of the population and can be generally classified into either pulsatile or non-pulsatile types ${ }^{[1]}$. The non-pulsatile form is recognised as the more common presentation of the two and is most often related to episodes of a continuous ringing or whistling sound ${ }^{[2]}$.

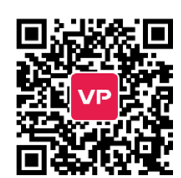


Pulsatile forms of tinnitus are the rarer manifestation of this condition, where the ringing symptoms experienced are associated with the vascular system ${ }^{[3]}$. Carotid stenosis is generally accepted as the most common cause of arterial pulsatile tinnitus ${ }^{[3]}$.

In current vascular surgery practice, tinnitus is very much correlated with the presence of distorted extracranial or intracranial blood vessels or to the presence of intracranial hypertension. Therefore, precluding an abnormally functioning auditory system. The heartbeat synchronous tinnitus is as we know a predominantly vascular pathology in origin and by in large can be diagnosed by different forms of angiography, including classical intravenous or intra-arterial angiography, computed tomography angiography, or more commonly magnetic resonance angiography.

Pulsatile tinnitus is typically subdivided into arterial heartbeat synchronous or venous "hum-like" pulsatile tinnitus ${ }^{[4]}$. It has been referred to as objective tinnitus. These perceived pulsations are most likely transmitted through the cerebrospinal fluid to the cochlea; a mechanism similar to what has been proposed as an explanation for bone conduction ${ }^{[5,6]}$.

Carotid artery atherosclerotic disease has been widely linked to tinnitus; however, reports emerging on stenotic subclavian, external carotid artery and reversal of blood flow in an aberrant occipital artery have also been implicated in the causality of pulsatile tinnitus ${ }^{[7-9]}$. The impact of flow disturbances in the carotid arteries on the inner ear has not yet been investigated thoroughly, and reports are very much limited by single-centre biased reporting. Direct meaning from such experiences has not been sufficient to bridge the gap in evidence in the literature. As the population is ageing, the standard techniques of audiology and oto-neurological testing is also a challenge to conduct in elderly populations, particularly in those with dementia. This coupled with a general absence of public awareness has not led to the introduction of generalised therapeutic interventions. These factors are likely to exacerbate the current health economic turmoil associated with the clinical care of this condition due to the surge of referrals to oto-neurological clinics and vascular surgery practices.

From a vascular surgery perspective and standpoint, carotid artery stenosis as a pre-existing cause for pulsatile tinnitus can be corrected with dilation and stenting or carotid endarterectomy ${ }^{[10,11]}$. Such intervention is believed to abolish the turbulent flow and ameliorate the clinical presentation allowing for a reasonable patient-related quality of life. However, it is currently unclear what clinical practice to implement due to the lack of clear evidence of best practice published in the literature. For example, ipsilateral carotid endarterectomy has been shown to effectively reduce if not eliminate pulsatile tinnitus in greater than $90 \%$ of patients with demonstrated intracranial carotid artery stenosis ${ }^{[12]}$. However, it is imperative to draw a meaningful conclusion from reported experiences and to be able to draw indications on when endarterectomy is to be favoured over the rather quantified approach of stenting. The location of the lesion is no longer sufficient to deduce the best applicable practice and more up to date guidelines are required for treating this disease.

This wide evidence-based literature review aims to describe the current understanding of the natural history and pathology of this condition in respect to carotid artery disease, as well as to compare the current therapeutic, clinical and surgical interventions utilised to allow clinicians and patients to make a well-informed decision in regard to their management plan. We also aim to outline the gap in existing evidence pertaining to this entity and to reflect on potentially different focuses for furthering evidencebased practice in this field.

\section{IS THERE A PHANTOM PERCEPTION BETWEEN CAROTID ARTERY DISEASE AND TINNITUS?}

Carotid artery stenosis may be asymptomatic or have subtle neurological presentation albeit of motor or sensory origin. Therefore, identifying a clinically significant carotid artery stenosis can be difficult to 
achieve in cases without symptoms or with the most minimal. On a parallel note, a reduction in cerebral blood flow may be a contributory factor and can potentially be the culpable weakest link.

Hence, to date, the standard diagnostic tool for delineating this is the widely available duplex ultrasonography. However, this tool is subjective and dependent on the operator. This raises the issue of potential subjective biases in reporting and utilization of this resource. Consequently, in an attempt to quantify the degree of stenosis present, other imaging modalities can also be implemented. These include classical angiography, computed angiography, and magnetic resonance angiography. However, those modalities raise a prudent question regarding timing and associated timely risk factors. The identification of these risk factors and the unequivocal management of Tinnitus, shown to have a vascular origin, can have an impact on central nervous system symptom manifestation and a natural effect on pulsatile tinnitus. The carotid intima-media thickness as delineated on the aforementioned imaging modalities can be of crucial help when identifiable in formulating the diagnosis and developing the axis of management but cannot surely affect or quantify the pulsatility.

The microvascular remodelling implicated in increased pulsatile tinnitus through the increased resistance in vascular beds and the emergence of microvascular ischemia has large implications on arterial stiffness and crucially on the success of any intervention whether surgical or endovascular.

\section{LITERATURE SEARCH STRATEGY}

Electronic searches were performed on a number of databases including PubMed, Scopus, Embase and Cochrane with no limits put in place on dates. Search terms included: natural history, carotid artery disease, and stenosis, lesion size, location, prevalence, and natural history, risk factors, survival rates, medical therapy, surgical intervention, and mortality. Search terms were charted to MeSH terms, combined using Boolean operations and also used as keywords. Papers were selected on the basis of their title and abstract. The reference lists of these selected papers were also reviewed to identify any relevant papers that might be suitable for inclusion for this study. Forty-five publications were eventually identified with dates ranging from 2002-2020. A full breakdown of number of studies identified is detailed in Figure 1.

\section{SELECTION CRITERIA}

Research papers were not excluded on the basis of their study design except for case reports. Any comments, editorials or opinions were not included for selection to provide an unbiased view. Papers were selected on the basis of providing primary endpoints of intervention used, the eventuality of the disease intervention and/or information regarding medical therapeutics, endarterectomy and angioplasty. Papers were not excluded on the basis of the age of the patient population.

\section{WHAT IS THE PREVALENCE CHARACTERISTICS AND PATHOPHYSIOLOGY OF TINNITUS IN VASCULAR SURGERY PRACTICE?}

To date, there is no prevailing consensus or guidelines that are sufficient to best describe the natural history of this disease and the current literature is lacking in detail regarding the prevalence and characteristics of the condition. Therefore, robust research into the pathophysiological factors, etiological mechanisms and pathogenesis of tinnitus in correlation with factors of carotid disease such as location, lesion size and extent of stenosis of affected vessels among others is required.

Historically, atherosclerosis within the carotids was credited as the main arterial cause of tinnitus. However, arterial stiffness has also become an important parameter that can predict the carotid event and can be correlated with the incidence of stroke $e^{[13]}$. 


\section{Databases searched: PubMed, Scopus, Cochrane, Embase}

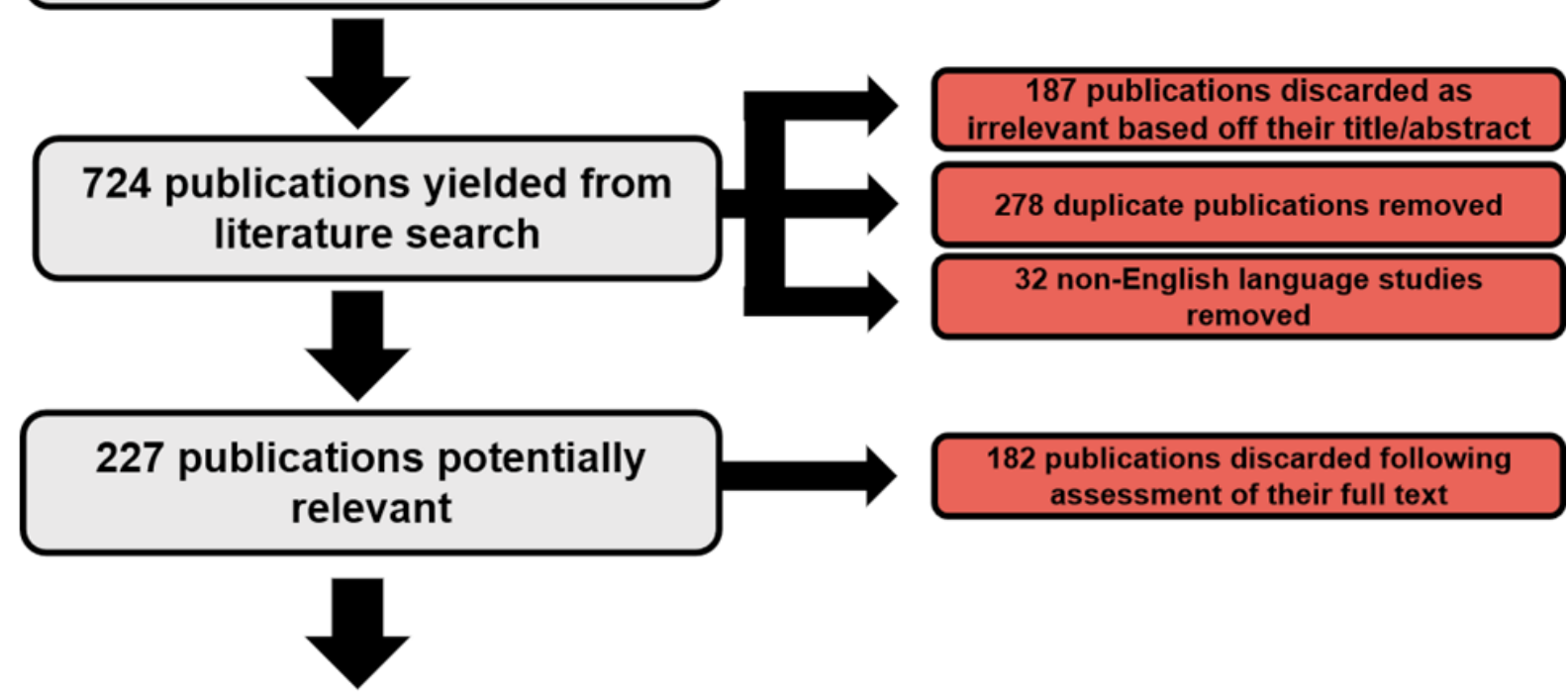

\section{5 publications deemed relevant}

Figure 1. Flow chart detailing steps undertaken in our extensive literature search

Arterial stiffness as a term is inclusive of vascular smooth muscle hyperplasia, an increase in collagen levels, degradation of elastin including fracture of the elastic lamellae, and intima-media thickening, thereby altering vascular smooth muscle cell tone, which over time results in the development of increased stiffness or arteriosclerosis ${ }^{[14]}$. Ageing, obesity, diabetes mellitus, and hyperlipidaemia are all factors relating to arterial stiffening ${ }^{[15]}$. Arterial stiffness, which can be measured as either aortic or carotid-femoral pulse wave velocity or through augmentation index, is now recognised as an important independent predictor of both future cardiovascular disease and all-cause mortality ${ }^{[16,17]}$. Augmentation index measures the central pulse pressure and is typically considered a more complex variable due to a combination of vascular elasticity and peripheral resistance ${ }^{[18]}$. An increase in aortic pulse wave velocity of 1 metre/second is correlated to an age, sex and risk factor adjusted increase of $14 \%$ for cardiovascular events and $15 \%$ for mortality ${ }^{[16]}$.

The cochlear microcirculation may be impaired by this increased arterial stiffness due to the terminal nature of this circulation. Yet, this emerged evidence did not yield a shred of conclusive pathophysiological evidence as to how it pertains to pulsatile tinnitus. It has been shown that increased arterial stiffness may lead to microvascular damage in the brain and this can manifest itself with a multitude of clinical symptoms and signs including tinnitus ${ }^{[19]}$. This had yielded to another entity related to carotid system arterial stiffness implicated in the development and severity of "idiopathic subjective tinnitus" ${ }^{\text {"[8] }}$.

Parameters utilized to support this concept from the studies ${ }^{[8,20]}$ that had recruited participants included serum lipid profile $(\mathrm{mg} / \mathrm{dL})$, pure-tone hearing, blood pressure $(\mathrm{mmHg})$, fasting glucose $(\mathrm{mg} / \mathrm{dL})$, and body mass index (BMI, $\mathrm{kg} / \mathrm{m}^{2}$ ). Hence, the common carotid artery stiffness index, common carotid intimamedia thickness, Young's elastic modulus, peak systolic velocity, end-diastolic velocity, resistive index, vessel diameter, pulsatility index, mean velocity and volume flow were measured in both the right and left common carotid arteries. 
These studies were able to intrinsically shed light on patient characteristics and the severity of the tinnitus. However, this was not uniformly applicable to both carotid artery disease, and independent predictors were unable to be constructed.

Another hypothesized scope outside of vascular surgery practice discussed the abnormal presence of neural activity inside the auditory system or tonotopic reorganization of the auditory cortex due to chronic deafferentation $^{[21]}$.

In relation to vascular medicine and surgery terms, this was transcribed to the etiological factors that may contribute to vascular hypoxia/ischemia, which have been considered as possible factors in the pathophysiology of tinnitus. However, this inconclusive hypothesis has not revealed any meaning to the cellular significance of vascular-related tinnitus and as such is irrelevant to pinpoint carotid system disease as a culpable factor to the occurrence of tinnitus. Brain-related peptide elements and circulation are far from being understood to conclude as an aforementioned hypothesis for this phenomenon.

Without scientifically led and clinically driven evidence, it is difficult to see how such a hypothesis can determine a correlation between carotid artery disease and tinnitus based on this particular evidence and much further study and exploration is needed to accept or negate this.

\section{OTO-NEUROSURGICAL EVIDENCE: A PROMISING \& INNOVATIVE APPROACH}

Recent advances in neuroimaging techniques, including positron emission tomography, magnetoencephalography, electroencephalography and functional magnetic resonance imaging, highlight the involvement of the central nervous system in the pathophysiological mechanism for tinnitus ${ }^{[22]}$. These neuroimaging investigations have shown that brain regions that are considered to be unconnected to the auditory system, including the anterior cingulate gyrus, amygdala, hippocampus, and parahippocampal regions are strongly indicated in the pathogenesis of subjective tinnitus. Utilizing resting-state functional magnetic resonance imaging (fMRI) in non-auditory brain regions, such as the rectus gyrus, thalamus, cingulate gyrus, inferior temporal gyrus, hippocampus, cerebellar hemisphere, caudate and medial superior frontal gyrus, demonstrated an association with tinnitus-related symptoms including depression, anxiety and loudness ${ }^{[23]}$.

However, studies surging on this front line were limited not only due to low numbers of control cases but also to a lack of quantification and vague anatomical correlation and orientation. Laureano et al. ${ }^{[24]}$, utilizing single-photon emission computerised tomography study with a sufficient number of control cases $(n=17$, sound methods of statistics and particularly precise anatomical orientation) were able to demonstrate that the cerebral blood flow in the left parahippocampus was significantly increased in tinnitus with normal hearing. This particular study intended to reconfirm the previous results of other resting-state fMRI studies using a different modality and method with intended benefit to identify ideal therapeutic targets for repetitive transcranial magnetic stimulation ${ }^{[24]}$.

Furthermore, attempts have been made to identify a different target for tinnitus and its correlation to carotid artery disease. Lesion size or location of carotid artery disease and the ability to combine these factors with arterial stiffness (using methods such as the carotid-femoral pulse wave velocity) and comparisons of local stiffness measurements (performed via Doppler ultrasonography) achieved conceptual accuracy in describing a meaningful mechanism with accurate values for tinnitus occurrence ${ }^{[12]}$. However, it is important to remain critical of emerging evidence, and as such, most of those studies had a small number of recruits and were unwarranted in determining the significant difference between the patient and control groups. 


\section{CAROTID ENDARTERECTOMY AN OLD APPROACH IN AN ENDOVASCULAR ERA AND THE VASCULAR-NEURO-PSYCHIATRIC AXIS}

By default, and without any consensus or attributable guidance, it is well-practiced in various multidisciplinary meetings and known that proximal lesions of carotid artery disease in the neck generally lend themselves to carotid endarterectomy practicing surgeons, while distal lesions get stratified and treated by angioplasty and stenting.

For years, intracranial carotid artery stenosis or carotid stenosis in the skull base have been generally treated using two approaches. One option, an initial balloon occlusion test performed under transcranial doppler and electroencephalogram monitoring can determine whether indeed the ipsilateral carotid artery can be sacrificed. If this is the case, one option is to ligate the symptomatic carotid artery and therefore arrest the turbulent flow. A second further option is to dilate and then stent the intracranial portion of the carotid artery, thereby resulting in a loss of arterial pulsatile tinnitus ${ }^{[5]}$. From these practices and reported experiences, more than $70 \%$ of patients with carotid stenosis-related tinnitus have immediate amelioration and relief of tinnitus ${ }^{[5]}$. Long-term and longitudinal studies correlating survivability and amelioration of symptoms are lagging.

Moreover, functional status and cognitive impact of these approaches are yet to be verified and studied in an unbiased format. As such, interventional as well as non-interventional medical procedures including carotid artery recanalization, appropriate pharmacological therapy and lifestyle modifications that are used to maintain blood flow in cerebral small vessels, are not well documented in any coherent evidence-based approach. This adds to the confounding gap in the literature and strictly limits our practice to surgeonoriented and not patient-centred care. It is interesting to note that tinnitus attributable to common carotid artery stenosis at its bifurcation has been historically treated by ligation or carotid endarterectomy ${ }^{[25]}$. However, curing this entity by endovascular stent angioplasty has received very little attention in the literature. As such, the heterogeneity of reporting and the lack of informal evidence of best medical practice reliability of results are questioned.

The advent but lack of availability of complex magnetic resonance imaging, in particular a combination of dynamic contrast-enhanced magnetic resonance imaging (MRI), dynamic susceptibility contrast MRI and blood oxygen level-dependent imaging MRI should be able to offer wide-ranging information on the effects of cerebral microcirculation on functional brain status and fluctuations of blood-brain barrier permeability. However, those modalities amongst others would not be in a position to add to studies with the concomitant neuropsychological assessment of patients with the carotid disease and post-tinnitus correction.

Recent advances leading to a new generation of stents has prompted clinicians to consider performing stent-assisted angioplasty as an alternative approach to angioplasty alone for intracranial stenosis ${ }^{[26,27]}$.

Cerebral blood flow, which can undergo assessment during computed tomography perfusion (CTP) examinations, improves after internal carotid artery stenting ${ }^{[28-30]}$. However, two further important CTPderived variables, blood-brain barrier permeability and mean transit time, are far less recognised in both the research and diagnosis of internal carotid stenosis.

Internal carotid artery stenosis-related brain hypoperfusion is generally associated with neurological, psychiatric, psychological and somatic deficits which can manifest with different variants of symptoms and clinical signs, including tinnitus. This can potentiate ill effect on health-related quality of life. To this effect, various studies have demonstrated that internal carotid stenosis without transient ischemic episodes 
or strokes, is detrimental to memory and executive functioning ${ }^{[31-34]}$. It has also been shown that cognitive function can be improved in symptomatic patients following internal carotid artery recanalization using both endarterectomy and carotid stenting ${ }^{[35,36]}$. Grunwald et al. ${ }^{[37]}$ have also shown cognitive improvements in both word fluency and in delayed recall when under neuropsychological testing that was conducted $24 \mathrm{~h}$ after internal carotid artery stenting in asymptomatic patients. Results in asymptomatic patients similar to those outlined above have also been published by Wang et al ${ }^{[30]}$ and Picchetto et al ${ }^{[38]}$.

To ameliorate the vascular-neuro-psychiatric axis as a causative element for tinnitus amongst other clinical manifestations, Suh et al. ${ }^{[39]}$ demonstrated that balloon expandable intracranial stent placement can be safely utilized in stable symptomatic patients. Adverse effect rates were far lower in the stable patient group $(4.1 \%)$ in comparison to the unstable patient group $(25.9 \%)$. Terada et al ${ }^{[40]}$ further published that stent placement is significantly more effective than percutaneous angioplasty for stenosis in the petrous or cavernous portion of the internal carotid and has low periprocedural morbidity (4.2\%). Goessens et al. ${ }^{[41]}$, demonstrated that internal carotid artery stenosis should be considered an independent risk factor for stroke. Rao et al ${ }^{[42]}$, Mathiesen et al. ${ }^{[43]}$ and Bakker et al. ${ }^{[44]}$ added to this bulk of evidence and stated that this contributes to the pathophysiology of depression and is also actually a risk factor for suicide in these stroke patients $^{[45]}$.

Therapeutic effects of stenting do exist and is has shown to be superior to that observed by medical prescriptions including selective serotonin reuptake inhibitors in these patient groups. However, in a study by Picchetto et al ${ }^{[38]}$, it was observed that there was no difference amongst pre- and post-scoring in the neuropsychological evaluation of both depression and anxiety carried out in asymptomatic internal carotid artery stenosis patients who received stenting.

Nonetheless, the approach and its desired effect on selective endovascular stenting for managing the ill effect of the vascular-neuro-psychiatric axis as a culpable cause for tinnitus has been demonstrated to be of promising value and with reliable results.

\section{CONCLUSION}

Therapeutic and surgical interventions amongst others such as classical endarterectomy or the surge of endovascular carotid stenting for the management of carotid artery disease-induced tinnitus may ameliorate this clinical condition, but their application is not without complications, compounded with a neurological disability or even death. No culpable evidence exists in the current era on what drives carotid artery stenosis and how this correlates with tinnitus. The exact pathophysiological mechanism is yet to be elucidated. The current reports of intervention are without meaningful and reliable evidence for managing this condition. Further studies of multicentre nature and well-constructed and powered trials are very much needed to build an evidence-based approach in an era of personalized and digital healthcare.

\section{DECLARATIONS}

\section{Authors' contributions}

Made substantial contributions to the conception and design of this study: Abdelhaliem A, Howard C, Bashir M, Elsantawy H, Al-Khaffaf $\mathrm{H}$

\section{Availability of data and materials}

Not applicable.

\section{Financial support and sponsorship}

None. 


\section{Conflicts of interest}

All authors declared that there are no conflicts of interest.

\section{Ethical approval and consent to participate}

Not applicable.

\section{Consent for publication}

Not applicable.

\section{Copyright}

(c) The Author(s) 2020.

\section{REFERENCES}

1. Bhatt JM, Lin HW, Bhattacharyya N. Prevalence, severity, exposures, and treatment patterns of tinnitus in the United States. JAMA Otolaryngol Head Neck Surg 2016;142:959-65.

2. Liu ZD, Fan ZM. Research progress of vascular pulsatile tinnitus. Lin Chung Er Bi Yan Hou Tou Jing Wai Ke Za Zhi 2017;31:1450-4.

3. Chari DA, Limb CJ. Tinnitus. Med Clin North Am 2018;102:1081-93.

4. Hofmann E, Behr R, Neumann-Haefelin T, Schwager K. Pulsatile tinnitus: imaging and differential diagnosis. Dtsch Arztebl Int 2013;110:451-8.

5. De Ridder D, Vanneste S, Menovsky T. Pulsatile tinnitus due to a tortuous siphon-like internal carotid artery successfully treated by arterial remodeling. Case Rep Otolaryngol 2013;2013:938787.

6. Han BI, Lee HW, Kim TY, Lim JS, Shin KS. Tinnitus: characteristics, causes, mechanisms, and treatments. J Clin Neurol 2009;5:11-9.

7. Conlin AE, Massoud E, Versnick E. Tinnitus: identifying the ominous causes. CMAJ 2011;183:2125-8.

8. Bayraktar C, Taşolar S. Relationship between increased carotid artery stiffness and idiopathic subjective tinnitus. Eur Arch Otorhinolaryngol 2017;274:2125-30.

9. Alexander MD, English J, Hetts SW. Occipital artery anastomosis to vertebral artery causing pulsatile tinnitus. J Neurointerv Surg 2014;6:e15

10. Hartung O, Alimi YS, Juhan C. Tinnitus resulting from tandem lesions of the internal carotid artery: combined extracranial endarterectomy and intrapetrous primary stenting. J Vasc Surg 2004;39:679-81.

11. Daneshi A, Hadizadeh H, Mahmoudian S, Sahebjam S, Jalesi A. Pulsatile tinnitus and carotid artery atherosclerosis. Int Tinnitus J 2004;10:161-4.

12. Huybrechts SA, Devos DG, Vermeersch SJ, Mahieu D, Achten E, et al. Carotid to femoral pulse wave velocity: a comparison of real travelled aortic path lengths determined by MRI and superficial measurements. J Hypertens 2011;29:1577-82.

13. Huang YS, Koo M, Chen JC, Hwang JH. The association between tinnitus and the risk of ischemic cerebrovascular disease in young and middle-aged patients: a secondary case-control analysis of a nationwide, population-based health claims database. PLoS One 2017;12:e0187474.

14. Nilsson PM, Boutouyrie P, Cunha P, Kotsis V, Narkiewicz K, et al. Early vascular ageing in translation: from laboratory investigations to clinical applications in cardiovascular prevention. J Hypertens 2013;31:1517-26.

15. Schiffrin EL. Vascular stiffening and arterial compliance. Implications for systolic blood pressure. Am J Hypertens 2004;17:39-48S.

16. Vlachopoulos C, Aznaouridis K, Stefanadis C. Prediction of cardiovascular events and all-cause mortality with arterial stiffness: a systematic review and meta-analysis. J Am Coll Cardiol 2010;55:1318-27.

17. Ben-Shlomo Y, Spears M, Boustred C, May M, Anderson SG, et al. Aortic pulse wave velocity improves cardiovascular event prediction: an individual participant meta-analysis of prospective observational data from 17,635 subjects. J Am Coll Cardiol 2014;63:636-46.

18. Mitchell GF, Hwang SJ, Vasan RS, Larson MG, Pencina MJ, et al. Arterial stiffness and cardiovascular events: the Framingham heart study. Circulation 2010;121:505-11.

19. Mustapha M, Nassir CMNCM, Aminuddin N, Safri AA, Ghazali MM. Cerebral small vessel disease (CSVD) - lessons from the animal models. Front Physiol 2019;10:1317.

20. Rafati M, Havaee E, Moladoust H, Sehhati M. Appraisal of different ultrasonography indices in patients with carotid artery atherosclerosis. EXCLI J 2017;16:727-41.

21. Eggermont JJ, Roberts LE. Tinnitus: animal models and findings in humans. Cell Tissue Res 2015;361:311-36.

22. Ueyama T, Donishi T, Ukai S, Yamamoto Y, Ishida T, et al. Alterations of regional cerebral blood flow in tinnitus patients as assessed using single-photon emission computed tomography. PLoS One 2015;10:e0137291.

23. Ueyama T, Donishi T, Ukai S, Ikeda Y, Hotomi M, et al. Brain regions responsible for tinnitus distress and loudness: a resting-state FMRI study. PLoS One 2013;8:e67778.

24. Laureano MR, Onishi ET, Bressan RA, Castiglioni ML, Batista IR, et al. Memory networks in tinnitus: a functional brain image study. PLoS One 2014;9:e87839.

25. Ihn YK, Jung WS, Kim BS. Disappeared pulsatile tinnitus related to petrous segment stenosis of the ICA after relief of the stenosis by 
stenting. Interv Neuroradiol 2013;19:97-101.

26. Yan Y, Wang J, Zhong C, Zhang Y, Wei Y, et al. Effects of endovascular stent-assisted angioplasty on cellular metabolism in the hippocampus of elderly patients with symptomatic vertebrobasilar artery stenosis. Med Sci Monit 2020;26:e922131.

27. Jin H, Wang J, Meng X, Li Y, Miao, Z, et al. safety of endovascular treatment for concomitant unruptured intracranial aneurysms and cerebral vascular stenosis. Chin Neurosurg J 2020; doi: 10.21203/rs.3.rs-40545/v1.

28. Szarmach A, Halena G, Kaszubowski M, Piskunowicz M, Studniarek M, et al. Carotid artery stenting and blood-brain barrier permeability in subjects with chronic carotid artery stenosis. Int J Mol Sci 2017;18:1008.

29. Frydrychowski AF, Winklewski PJ, Szarmach A, Halena G, Bandurski T. Near-infrared transillumination back scattering sounding--new method to assess brain microcirculation in patients with chronic carotid artery stenosis. PLoS One 2013;8:e61936.

30. Trojanowska A, Drop A, Jargiello T, Wojczal J, Szczerbo-trojanowska M. Changes in cerebral hemodynamics after carotid stenting: evaluation with CT perfusion studies. J Neuroradiol 2006;33:169-74.

31. Wang T, Sun D, Liu Y, Mei B, Li H, et al. The impact of carotid artery stenting on cerebral perfusion, functional connectivity, and cognition in severe asymptomatic carotid stenosis patients. Front Neurol 2017;8:403.

32. Romero JR, Beiser A, Seshadri S, Benjamin EJ, Polak JF, et al. Carotid artery atherosclerosis, MRI indices of brain ischemia, aging, and cognitive impairment: the Framingham study. Stroke 2009;40:1590-6.

33. Sztriha LK, Nemeth D, Sefcsik T, Vecsei L. Carotid stenosis and the cognitive function. J Neurol Sci 2009;283:36-40.

34. Martinić-Popović I, Lovrenčić-Huzjan A, Demarin V. Advanced asymptomatic carotid disease and cognitive impairment: an understated link? Stroke Res Treat 2012;2012:981416.

35. Yamashita T, Ogasawara K, Kuroda H, Suzuki T, Chida K, et al. Combination of preoperative cerebral blood flow and 123I-iomazenil SPECT imaging predicts postoperative cognitive improvement in patients undergoing uncomplicated endarterectomy for unilateral carotid stenosis. Clin Nucl Med 2012;37:128-33.

36. Chida K, Ogasawara K, Suga Y, Saito H, Kobayashi M, et al. Postoperative cortical neural loss associated with cerebral hyperperfusion and cognitive impairment after carotid endarterectomy: 123I-iomazenil SPECT study. Stroke 2009;40:448-53.

37. Grunwald IQ, Papanagiotou P, Struffert T, Politi M, Krick C, et al. Reversal of flow during carotid artery stenting: use of the Parodi antiembolism system. Neuroradiology 2007;49:237-41.

38. Picchetto L, Spalletta G, Casolla B, Cacciari C, Cavallari M, et al. Cognitive performance following carotid endarterectomy or stenting in asymptomatic patients with severe ICA stenosis. Cardiovasc Psychiatry Neurol 2013;2013:342571.

39. Suh DC, Kim JK, Choi JW, Choi BS, Pyun HW, et al. Intracranial stenting of severe symptomatic intracranial stenosis: results of 100 consecutive patients. AJNR Am J Neuroradiol 2008;29:781-5.

40. Terada T, Tsuura M, Matsumoto H, Masuo O, Yamaga H, et al. Endovascular surgery for internal carotid stenoses. Results of PTA vs. stenting. Interv Neuroradiol 2000;6 Suppl 1:233-5.

41. Goessens BM, Visseren FL, Kappelle LJ, Algra A, van der Graaf Y. Asymptomatic carotid artery stenosis and the risk of new vascular events in patients with manifest arterial disease: the SMART study. Stroke 2007;38:1470-5.

42. Rao AS, Makaroun MS, Marone LK, Cho JS, Rhee R, et al. Long-term outcomes of internal carotid artery dissection. J Vasc Surg 2011;54:370-4; discussion 375 .

43. Mathiesen EB, Waterloo K, Joakimsen O, Bakke SJ, Jacobsen EA, et al. Reduced neuropsychological test performance in asymptomatic carotid stenosis: the Tromsø study. Neurology 2004;62:695-701.

44. Bakker FC, Klijn CJ, Jennekens-Schinkel A, Kappelle LJ. Cognitive disorders in patients with occlusive disease of the carotid artery: a systematic review of the literature. J Neurol 2000;247:669-76.

45. Lovett JK, Rothwell PM. Suicide in a patient with symptomatic carotid occlusion. J R Soc Med 2002;95:93-4. 